\title{
Philosophiques
}

\section{Intentions rationnelles et acceptations en délibération}

\section{Olivier Roy}

Volume 35, numéro 2, automne 2008

URI : https://id.erudit.org/iderudit/000441ar

DOI : https://doi.org/10.7202/000441ar

Aller au sommaire du numéro

Éditeur(s)

Société de philosophie du Québec

ISSN

0316-2923 (imprimé)

1492-1391 (numérique)

Découvrir la revue

Citer cet article

Roy, O. (2008). Intentions rationnelles et acceptations en délibération. Philosophiques, 35(2), 525-545. https://doi.org/10.7202/000441ar

\section{Résumé de l'article}

Dans cet article, je montre que quatre normes de rationalité associées aux intentions peuvent être déduites de normes similaires s'appliquant aux acceptations en contextes délibératifs, un type d'état mental apparenté mais irréductible aux croyances par lequel un agent tient certains faits pour acquis lorsqu'il délibère. Je montre que cette approche, que je nomme le pragmatisme hybride, évite certaines limitations de l'approche la plus prisée dans la littérature, le cognitivisme, et qu'en comparaison avec les approches purement pragmatistes, principales rivales du cognitivisme, le pragmatisme hybride rend mieux justice à nos intuitions relatives aux normes associées aux intentions. Je montre enfin que le pragmatisme hybride permet d'expliquer comment les intentions influencent le raisonnement pratique, et de ce fait, comblent un vide important dans les théories contemporaines. 


\title{
Intentions rationnelles et acceptations en délibération
}

\author{
OLIVIER ROY
}

Faculté de Philosophie, Université de Groningen (Pays-Bas)

o.roy@rug.nl

\begin{abstract}
RÉSUMÉ. - Dans cet article, je montre que quatre normes de rationalité associées aux intentions peuvent être déduites de normes similaires s'appliquant aux acceptations en contextes délibératifs, un type d'état mental apparenté mais irréductible aux croyances par lequel un agent tient certains faits pour acquis lorsqu'il délibère. Je montre que cette approche, que je nomme le pragmatisme hybride, évite certaines limitations de l'approche la plus prisée dans la littérature, le cognitivisme, et qu'en comparaison avec les approches purement pragmatistes, principales rivales du cognitivisme, le pragmatisme hybride rend mieux justice à nos intuitions relatives aux normes associées aux intentions. Je montre enfin que le pragmatisme hybride permet d'expliquer comment les intentions influencent le raisonnement pratique, et de ce fait, comblent un vide important dans les théories contemporaines.
\end{abstract}

\begin{abstract}
In this paper, I show that four normative requirements on rational intentions follow from similar requirements on acceptations in deliberative contexts, a type of mental state close but irreducible to beliefs by which agents take some facts for granted in deliberation. I show that this approach, which I call hybrid pragmatism, avoid shortcomings of cognitivism, one of the most prominent one in the literature. I show furthermore that in comparison with purely pragmatist approaches, the main rivals of cognitivism, an hybrid form of pragmatism makes better justice to our intuitions regarding the sources of the normative requirements on rational intentions, and that it allows for understanding how intentions influence practical reasoning.
\end{abstract}

\section{Introduction}

Bien qu'il existe un consensus relativement large en philosophie de l'action sur le fait que les intentions, pour être dites rationnelles, doivent satisfaire certaines contraintes, peu d'auteurs s'entendent sur le pourquoi de ces contraintes. À la suite de M. Bratman (à paraître), on peut distinguer, dans la littérature récente, deux grands types d'expli-cations des normes de rationalité associées aux intentions. D'un côté, les explications cognitivistes tentent de déduire ces normes à partir de normes similaires s'appliquant aux croyances (G. Harman (1976) et (1986), R. J. Wallace (2003) et (2006), J. D. Velleman (2003) et (2007) et K. Setiya (2007) et (à paraître)). De l'autre, les tenants d'une approche pragmatiste, tout particulièrement Bratman (à paraître), rejettent ce recours au domaine des croyances et tentent de déduire ces normes à partir de principes pratiques généraux. 
Dans cet article j'explore une approche originale de la justification des contraintes normatives associées aux intentions, que je nomme le pragmatisme bybride, se basant sur le concept d'acceptation en contexte délibératif ${ }^{1}$. Je montre que cette approche permet de dériver lesdites contraintes normatives, d'une manière qui fait à la fois justice aux intuitions fortes motivant le cognitivisme et le pragmatisme, tout en évitant certaines difficultés auxquelles ces approches font face.

L'article se développe comme suit. Dans la section 2, je présente les normes de rationalité qui devront être déduites par la suite. Je motive ensuite, dans la section 3, la recherche d'une approche alternative au cognitivisme en montrant certaines de ses limitations importantes. Je jette les bases de cette approche alternative dans la section 4 . Je présente à tour de rôle les notions d'acceptations en contextes délibératifs, d'arrière-plan cognitif adapté ${ }^{2}$ et de pertinence pour une délibération. J'examine ensuite trois normes de rationalité régissant l'arrière-plan cognitif adapté et les acceptations. Je développe l'approche pragmatiste hybride dans la section 5, en en présentant tout d'abord le postulat fondamental, puis en montrant qu'avec ce dernier on arrive à dériver les normes de rationalité associées aux intentions. En fin de section, je présente quelques arguments plus généraux en faveur de cette approche. Je montre qu'elle permet de comprendre comment les intentions influencent le raisonnement pratique, une question importante mais jusqu'à présent laissée en plan dans la littérature. Je soutiens également qu'elle trouve une justification normative pragmatiste plus unifiée que le cognitivisme, et qu'elle rend mieux justice que le pragmatisme pur à nos intuitions quant à la nature théorique de certaines des normes s'appliquant aux intentions.

\section{Quatre contraintes de rationalité associées aux intentions}

La plupart des théories contemporaines des intentions acceptent l'idée que ces états, pour être dits rationnels, doivent satisfaire les contraintes normatives suivantes.

Cohérence moyens-fin: Un agent ayant l'intention d'accomplir une certaine action doit avoir l'intention de faire ce qu'il croit nécessaire pour accomplir cette action, ou doit, à tout le moins, avoir l'intention de former ladite intention en temps et lieu.

Ici les moyens sont entendus comme nécessaires pour autant que leur réalisation dépende du fait que l'agent ait explicitement l'intention de les accomplir. Un agent ayant l'intention d'allumer la lumière n'est pas tenu de former une intention relative au passage du courant dans le circuit électrique de la pièce même si, dans des circonstances normales, cela est nécessaire à la réalisation de cette intention.

1. Je traduis ici l'expression anglaise "acceptances in contexts of deliberations".

2. Je traduis ici l'expression «adjusted cogniti ground» de Bratman (1991). 
Les cas d'incohérence moyens-fin qui seront discutés dans la suite mettront en jeu une incomplétude plutôt qu'une contradiction directe dans la structure intentionnelle de l'agent. Il s'agit de cas où l'agent a l'intention de réaliser une fin $\mathrm{F}$, croit que pour réaliser $\mathrm{F}$ il doit former l'intention de réaliser un des moyens dans l'ensemble $\mathrm{M}$, mais ne forme pas cette intention. Dans une forme plus forte d'incohérence moyens-fin, l'agent formerait tout simplement une intention excluant la réalisation de $\mathrm{M}$. Cette seconde forme plus forte d'incohérence peut être réduite à une violation de la norme d'agglomération ciblée, que je présente à l'instant, et pour cette raison je me concentrerai sur les cas d'incohérence provenant d'une incomplétude.

Cohérence avec les croyances ${ }^{3}$ : Les intentions d'un agent doivent être réalisables dans un monde où l'ensemble de ses croyances s'avèrent, en assumant que ces dernières sont cohérentes entre elles. L'agent ne doit pas former d'intentions à propos d'actions qu'il croit, en outre, impossibles à réaliser. Il est à noter que l'agent n'est pas tenu de croire que ses intentions sont réalisables.

Cohérence interne: Cette contrainte prend deux formes. En premier lieu, l'agent est tenu de ne pas former d'intentions dont le contenu est contradictoire, par exemple l'intention de faire l'action A et son contraire. J'appellerai cette contrainte la cohérence $d u$ contenu des intentions. Certains auteurs vont cependant plus loin et demandent que les intentions d'un agent ne se contredisent pas entre elles; que lorsqu'un agent a l'intention de faire A et l'intention de faire $\mathrm{B}$, la réalisation de la première n'exclut pas la réalisation de la seconde. J'appellerai cette contrainte la cohérence mutuelle des intentions. Un ensemble d'intentions peut évidemment être mutuellement incohérent sans qu'aucun de ses éléments n'ait un contenu incohérent.

Agglomération ${ }^{4}$. Suivant Yaffe (2004, p. 511-512), on peut distinguer deux lectures du principe d'agglomération dans la littérature.

La première, que j'appellerai agglomération systématique, stipule qu'un agent ne peut rationnellement avoir simultanément l'intention de faire A et l'intention de faire B sans avoir l'intention de faire A et B. Comme son nom l'indique, la rationalité des intentions d'un agent passe, selon ce principe, par une agglomération systématique de ces dernières, quelles qu'elles soient et peu importe leur relation.

Yaffe (idem) a soutenu, de manière convaincante selon moi, que la norme d'agglomération systématique est trop forte pour être considérée comme une exigence réelle s'appliquant aux intentions rationnelles, et il propose plutôt une lecture plus faible de cette norme, que j'appellerai l'agglomération ciblée. Selon ce principe, il est irrationnel d'avoir simultanément l'intention de faire $\mathrm{A}$ et l'intention de faire $\mathrm{B}$ si l'intention de faire

3. J'adapte ici l'expression anglaise «strong belief consistency» des travaux de Bratman (1987). Dans la suite, je traduirai de même le terme anglais "consistency" par "cohérence".

4. Je traduis ici directement le terme technique "agglomerativity». Voir Bratman (à paraître) et Velleman (2003). 
A et $\mathrm{B}$ contrevenait à d'autres normes de rationalité associées aux intentions. L'agglomération ciblée n'exige donc pas de combiner systématiquement toutes les intentions d'un agent. Cela ne leur est demandé que lorsque cet exercice «met au jour des tensions, lorsqu'il y a doutes quant à la rationalité de l'intention conjonctive $e^{5}$. Les tensions en question concernent ici «d'autres normes d'intentions rationnelles ${ }^{6}$.»

L'agglomération ciblée tire donc sa force normative des autres normes de rationalité présentées plus haut. L'intention de faire A et l'intention de faire son contraire, par exemple, violent la norme d'agglomération ciblée parce que l'intention conjointe, celle de faire A et son contraire, viole la norme de cohérence interne des intentions. Comme cet exemple le suggère, la norme de cohérence mutuelle des intentions devient, de ce point de vue, un cas particulier de la norme d'agglomération ciblée.

Dans la suite, je ne considérerai que l'agglomération ciblée, et laisserai donc de côté l'agglomération systématique. Non seulement peut-on douter du fait que cette dernière soit réellement une norme de rationalité associée aux intentions, mais elle pose aussi des problèmes particuliers tant pour le cognitivisme que pour l'approche en termes d'acceptation que j'explorerai plus loin. (Le lecteur intéressé peut consulter Bratman (à paraître) et Roy (2008, chap. 6) pour plus de détails.)

Nous avons donc quatre normes s'appliquant aux intentions: la cohérence moyens-fin, la cohérence avec les croyances, la cohérence du contenu et l'agglomération ciblée. Je me tourne maintenant vers l'explication cognitiviste de ces normes.

\section{Cognitivisme, approche et limites}

Je présente ici brièvement la forme de cognitivisme qui sera la plus importante dans la suite, et explique pourquoi elle échoue à expliquer de manière satisfaisante les normes tout juste présentées. Je n'entrerai pas dans le détail de l'argumentaire cognitiviste, et ne discuterai ni les diverses formes de cette approche ni le concept fonctionnaliste de croyance qui les sous-tend. Le lecteur intéressé par cette discussion peut consulter Roy (2008, pp. 157167). Pour motiver le développement d'une approche alternative, il est ici suffisant de mentionner les problèmes importants de l'approche cognitiviste.

L'entreprise cognitiviste consiste à montrer que les normes de rationalité associées aux intentions peuvent être dérivées de normes de rationalité similaires associées aux croyances, soit la cohérence interne des croyances, l'agglomération systématique et la cohérence explicative ${ }^{7}$. Cette déduction repose sur

5. Traduction libre de "makes conflicts evident to themselves, when there is doubt as to the rationality of the conjunctive intentions". Yaffe (idem).

6. Traduction libre de «with other norms of rational intentions». Yaffe (idem).

7. Voir à nouveau Roy (2008, pp. 157-167) pour une présentation détaillée de ces normes. 
un lien plus ou moins fort postulé entre intentions et croyances, dont le plus utilisé dans la littérature est ce que je nomme le cognitivisme moyen ${ }^{8}$.

\section{(PCM) Avoir l'intention de faire A implique croire que l'on fera A.}

L'idée principale derrière le cognitivisme moyen est, avec une théorie fonctionnaliste des croyances en arrière-plan, qu'avoir l'intention de faire A implique avant tout être disposé à inclure, dans les raisonnements théoriques et pratiques, le fait que l'action $\mathrm{A}$ sera accomplie, et ce, d'une manière qui reste ouverte aux ajustements à la lumière de nouvelles évidences. En particulier, ce postulat exclut qu'un agent puisse considérer possible que l'action A ne soit pas accomplie?.

Le cognitivisme moyen est en mesure de dériver la cohérence interne des intentions, celle de cohérence avec les croyances, ainsi que l'agglomération ciblée. Il arrive également à dériver la cohérence moyens-fin, mais seulement en faisant appel à une norme de rationalité pratique sur laquelle je reviendrai plus loin. Ce recours sape une importante motivation derrière le cognitivisme, soit de montrer que les normes de rationalité pratiques associées aux intentions sont, en fin de compte, explicables dans les seuls termes de la rationalité théorique.

À elle seule, cette difficulté justifie la quête d'approches alternatives pour l'explication des normes de rationalité associées aux intentions. S'ajoute à cela le fait que le postulat cognitiviste moyen est lui-même objet de controverse. Par exemple, selon Bratman (1987, p. 37-39) et (à paraître, p. 3), il est contre-intuitif de supposer qu'avoir l'intention de faire A implique avoir la croyance que l'on fera A, parce que cela oblige à conclure qu'avoir l'intention de faire A implique être disposé à inclure le fait que l'on fera A dans des raisonnements théoriques ultérieurs, conclusion qui semble trop forte ${ }^{10}$.

À la lumière de la difficulté rencontrée par la dérivation des normes s'appliquant aux intentions et des doutes que l'on peut avoir envers le postulat cognitiviste moyen, il semble d'autant plus pertinent d'explorer de nouvelles

8. En comparaison, on nommera " postulat cognitiviste faible » la thèse selon laquelle avoir l'intention de faire A implique croire qu'il est possible de faire A, et "postulat cognitiviste fort » la thèse selon laquelle avoir l'intention de faire A se réduit à un type particulier de croyance à propos du fait que l'on fera A. Le postulat cognitiviste moyen fut défendu par Harman (1986). Wallace (2003 et 2006) est un des principaux tenants du cognitivisme faible. Le cognitivisme fort est, quant à lui, le plus populaire dans la littérature, étant défendu par Harman (1976), Velleman (2007) et Setiya (2007). J'ai noté dans le texte que le postulat cognitiviste moyen est le "plus utilisé " parce même les dérivations basées sur la version forte du postulat cognitiviste n'utilisent, en fin de compte, que l'implication des intentions vers les croyances.

9. Voir Schwitzgebel (2006) et Shah et Velleman (2005, p. 2-3) pour la définition fonctionnaliste du concept de croyance que j'utilise ici

10. Je justifie cette interprétation de l'argument de Bratman dans (Roy, 2008, pp. 150151). 
stratégies d'argumentation. C'est ce que je fais dans la prochaine section, en invoquant le concept d'acceptation en contextes délibératifs.

\section{Acceptations en contextes délibératifs}

Dans cette section, je jette les bases conceptuelles sur lesquelles s'édifiera l'argument dans la section suivante. Je présente le concept d'acceptation en contextes délibératifs, ou simplement d'acceptation en délibérations, la notion d'arrièreplan cognitif adapté qui lui est complémentaire, et dresse une liste de contraintes normatives s'appliquant à ce dernier. En cours de route, je serai amené à discuter du principe de pertinence pour une délibération.

\subsection{Acceptation en délibération}

Nombre de philosophes ont remarqué que la délibération pratique met en jeu des états cognitifs proches mais différents des croyances, états qui consistent à tenir certains faits pour acquis, ou à accepter certains faits pour les besoins de la délibération ${ }^{11}$.

Les acceptations en délibérations sont généralement vues comme des attitudes consistant à tenir certains faits pour établis d'une manière qui, comme pour les croyances, est réglée sur le vrai et s'ajuste à l'apport de nouvelles évidences $^{12}$ mais, contrairement à ces dernières, est également " réglée sur la pratique » et s'ajuste aussi aux «exigences pragmatiques » ${ }^{13}$. Plus précisément, je considérerai l'acceptation que $\mathrm{P}$ dans une délibération donnée comme une attitude consistant à prendre $\mathrm{P}$ pour vrai dans cette délibération à la lumière soit d'évidences en faveur de P, soit de considérations pratiques liées au contexte délibératif en question, et qui est justifiée seulement dans les cas où soit $\mathrm{P}$ est vrai, soit l'acceptation de $\mathrm{P}$ est avantageuse d'un point de vue pratique ${ }^{14}$.

11. La présentation, dans cette section, se base principalement sur l'étude de Bratman (1991). On trouve des considérations similaires, quoique divergentes sur certains points, dans Grice (1971), Cohen (1989) et Engel (1998). Holton (1994) a également discuté une notion appa-rentée aux acceptations, dans le cadre d'une étude sur le concept de relation de confiance. L'idée de tenir certains faits pour acquis dans le contexte de la théorie des intentions figure déjà dans les travaux d'Harman (1976), quoique Williams (1973) soit, à ma connaissance, le premier à avoir utilisé le terme «acceptance» dans le sens où je l'entends ici. Holton (idem) et Alonso (2008) discutent aussi la notion d'acceptation, mais en utilisant une terminologie quelque peu différente : «reliance " plutôt qu' «acceptance». Notons enfin que Shah et Velleman (2005) utilisent aussi le terme "acceptance ", non pas pour dénoter l'attitude de tenir certains faits pour acquis en contextes délibératifs, mais plutôt pour référer à l'attitude générale de tenir une proposition pour vraie. Croyances, suppositions et acceptations en délibérations sont toutes des sous-catégories de ce qu'ils nomment "acceptances".

12. Voir encore une fois Shah et Velleman (2005, p. 2-3), pour une description des croyances en termes similaires.

13. Traduction libre de "regulated for practice» et «pragmatic considerations» (Alonso, 2008).

14. Il y a évidemment matière à débat sur le poids relatif des considérations pratiques et factuelles pouvant justifier une acceptation. Dans ce qui suit, je laisserai cependant cette question en plan parce qu'il est suffisant de compte le fait que les acceptations peuvent être jugées correctes pour l'un des deux types de raisons. Voir Alonso (2008), pour une discussion détaillée. 
Les acceptations se distinguent ainsi des croyances dans la manière dont elles sont engendrées et dans leur critère d'évaluation. J'adopte ici la caractérisation de Shah et Velleman (2005) selon laquelle un état ne peut compter comme une croyance s'il est engendré, révisé ou abandonné à la lumière d'autres considérations que l'apport de nouvelles évidences ou la conclusion de raisonnements théoriques. De même, une croyance adoptée pour des raisons pratiques, pensons aux cas où un agent "prend ses rêves pour la réalité " ${ }^{15}$, sera jugée comme irrationnelle, alors que c'est le propre des acceptations que de prendre place dans une délibération en réponse à de telles contraintes non fondées sur l'évidence. Il est à noter que c'est la valeur pratique de l'acceptation en elle-même qui peut entrer en ligne de compte lors de la justification, et non les potentiels bienfaits qu'aurait pour l'agent la réalisation du contenu de cet état mental.

Cette sensibilité aux contraintes pragmatiques des délibérations explique un autre point important de divergence entre acceptations et croyances : le fait qu'elles soient dépendantes des contextes délibératifs dans lesquels elles apparaissent. Il n'est ni contradictoire ni irrationnel pour un agent de tenir pour acquis que $\mathrm{P}$ dans une délibération et de ne pas accepter ce fait dans une autre, simplement parce les conditions pratiques entourant la première et la seconde diffèrent. Pour les croyances, au contraire, il semble irrationnel pour un agent de croire que $\mathrm{P}$ dans un contexte et de ne pas croire que $\mathrm{P}$ dans un autre si les deux contextes sont équivalents en ce qui concerne l'évidence en faveur de P. Seul l'apport d'évidences empiriques ou théoriques justifient la révision d'une croyance, alors qu'un changement d'acceptation peut être provoqué par un changement dans les contraintes pratiques d'une délibération.

Notons enfin que les acceptations sont mises en jeu dans la délibération par une décision de fixer comme vraies ou de suspendre ${ }^{16}$ certaines croyances. Par «fixer comme vraies ", j'entends ici accepter comme un fait établi quelque chose à propos de quoi on n'est pas absolument certain, et vice-versa pour la suspension : ne pas accepter un fait à propos duquel on est par ailleurs certain. Dans les deux cas, ce processus se distingue de la formation normale des croyances en ce qu'il est la plupart du temps volontaire et qu'il peut, comme nous l'avons vu, légitimement faire intervenir des considérations pratiques.

\subsection{L'arrière-plan cognitif adapté aux délibérations}

La présence d'acceptations en contextes délibératifs révèle que l'arrière-plan cognitif, c'est-à-dire ce que l'agent tient pour vrai lors d'une délibération, diffère de l'arrière-plan cognitif par défaut de l'agent, que j'entends ici comme l'ensemble de ses croyances. Bratman (1991, p. 29) utilise l'adjectif " adapté ${ }^{17}$

15. J'utilise ici la traduction de wishful thinking qui me fut proposée par N. Kaufmann.

16. Je traduis ici les expressions «positing» et «bracketing» de Bratman (1991, p. 29).

17. Je traduis ici "adjusted background of deliberation" et plus haut «default cognitive background». 
pour marquer cette différence. L'arrière-plan cognitif des délibérations est adapté justement parce qu'il met en jeu des acceptations introduites par fixation ou suspension de croyances présentes dans l'arrière-plan cognitif par défaut. Cela n'exclut pas que des croyances intactes, c'est-à-dire n'ayant pas été fixées ou suspendues, prennent place dans l'arrière-plan ajusté d'une délibération. Ce dernier peut très bien contenir à la fois des croyances et des acceptations.

Comme la fixation ou la suspension volontaire de certaines croyances est un processus qui demande temps et énergie, on ne peut simplement supposer que toutes les croyances de l'agent, ajustées ou non, figurent dans l'arrièreplan cognitif des délibérations, ni même supposer qu'elles devraient toutes y figurer. J'adopterai ici la position de Bratman (1991, 30), selon laquelle une croyance est comptée comme faisant partie d'une délibération si elle y est pertinente et que l'agent ne l'a pas déjà explicitement fixée ou suspendue dans cette délibération. Je discuterai sous peu la notion de pertinence pour une délibération, mais pour l'instant il suffit de noter qu'elle est mise en jeu pour limiter le nombre de croyances qui comptent comme figurant dans un contexte délibératif donné. Sans elle toutes les croyances qui ne sont pas explicitement fixées ou suspendues seraient automatiquement importées dans l'arrièreplan cognitif d'une délibération. De par le filtre de pertinence, l'arrière-plan cognitif adapté des délibérations peut laisser indéterminés la vérité ou le degré de confiance de l'agent à propos de certains faits.

\subsection{Pertinence pour une délibération}

La notion de pertinence pour une délibération est non seulement importante pour éviter une surcharge de l'arrière-plan cognitif adapté, mais comme nous le verrons plus loin, elle est aussi au cœur de la dérivation en termes d'acceptations des normes de rationalité associées aux intentions.

Je considérerai qu'une croyance que $P$ est pertinente pour un contexte délibératif si son inclusion dans l'arrière-plan cognitif adapté change de manière significative le résultat de cette délibération, de sorte que la décision prise en prenant en compte que $\mathrm{P}$ est significativement différente de celle prise en ne prenant pas $\mathrm{P}$ en compte, ou si elle concerne directement le contenu d'une intention prise en compte dans cette même délibération. Pour les intentions, je considérerai qu'une intention de faire A est pertinente pour un contexte délibératif donné si accepter que l'on fera A dans cette délibération en change significativement l'issue, c'est-à-dire si accepter que l'on fera $\mathrm{A}$ dans une délibération mène à une décision différente de celle à laquelle on parviendrait s'il l'on ne tenait pas ce fait pour acquis ${ }^{18}$.

Je ne préciserai pas davantage la notion de pertinence parce que, dans la suite, il sera suffisant de pouvoir discerner les cas extrêmes de pertinence et d'impertinence, qu'une compréhension intuitive de ce concept dans l'esprit

18. Voir par exemple les cas de «surcharge » (overloading) examinés dans (Pollack, 1991). 
des critères tout juste avancés semble en mesure de faire. Ces critères impliquent, en particulier, que l'on ne peut considérer que toute croyance est pertinente pourvu qu'elle puisse avoir une quelconque conséquence, si petite soit-elle, sur le résultat d'une délibération. Beaucoup trop de croyances seraient jugées pertinentes sous ce critère, d'où l'idée d'influence significative.

Le fait que je ne développe pas de manière plus précise le concept de pertinence pour une délibération ni celui d'influence significative ne signifie pas que ces concepts soient d'une faible importance pour l'explication des normes de rationalité associées aux intentions, bien au contraire. Définir les contours précis de ces notions est une tâche importante, et sans aucun doute difficile, qui va cependant au-delà des ambitions de cet article, et que la présente analyse peut, dans une large mesure, laisser de côté.

\subsection{Contraintes normatives sur l'arrière-plan cognitif adapté}

Tout comme dans l'argumentaire cognitiviste, la dérivation des normes de rationalité associées aux intentions que j'examine plus bas se base sur un certain nombre de normes de rationalité similaires associées non pas aux croyances, ou plus généralement à l'arrière-plan cognitif par défaut, mais à l'arrière-plan cognitif adapté des délibérations et aux acceptations. La dérivation utilisera plus précisément trois normes : la fermeture sous la conséquence logique, la cohérence interne et la cohérence explicative.

Fermeture sous la conséquence logique. Il sera supposé que l'arrière-plan adapté des délibérations doit être fermé sous la conséquence logique, au sens limité où il est fermé sous l'implication matérielle classique et sous la conjonction. $\mathrm{Si}$ « $\mathrm{P}$ » et «si $\mathrm{P}$ alors $\mathrm{Q}$ » figurent dans l'arrière-plan adapté d'une délibération d'un certain agent, alors " $Q$ » devrait y figurer également. De même manière, si « $\mathrm{P}$ » et « $\mathrm{Q}$ » figurent dans l'arrière-plan cognitif adapté alors «P et $\mathrm{Q}$ » devrait y figurer également.

La fermeture sous la conséquence logique implique l'agglomération systématique des éléments de l'arrière-plan adapté, mais d'une manière qui est restreinte aux contextes délibératifs. Si un agent accepte que $\mathrm{P}$ et accepte que $\mathrm{Q}$ dans un certain contexte, il est sommé d'accepter que $\mathrm{P}$ et $\mathrm{Q}$, mais seulement dans ce contexte, et ce, même si l'un des deux éléments de la conjonction provient directement de l'arrière-plan cognitif par défaut, sans avoir été fixé ni suspendu. En comparaison, l'agent est tenu à ses croyances agglomérées dans l'arrière-plan par défaut, et ce, indépendamment du contexte où elles apparaissent.

Notons que cette contrainte augmente considérablement le nombre de faits figurant dans l'arrière-plan cognitif adapté des délibérations, mais qu'elle n'implique pas que ce dernier fixe la vérité de tous les faits possibles.

Cohérence interne des intentions. Tout comme les intentions, il sera supposé ici que le contenu des éléments de l'arrière-plan cognitif adapté aux délibérations ne doit pas être contradictoire. Il est à noter qu'en présence de la norme de fermeture sous la conséquence logique, la norme de cohérence 


\section{$534 \cdot$ Philosophiques / Automne 2008}

interne implique une forme restreinte de cohérence des acceptations avec les croyances, au sens où ce qui est tenu pour acquis ne doit pas contredire les croyances directement importées dans l'arrière-plan cognitif. La portée précise de cette contrainte de cohérence avec les croyances dépend bien entendu du nombre de celles qui se verront transportées de l'arrière-plan par défaut vers l'arrière-plan cognitif, et donc de la notion de pertinence pour une délibération que j'ai brièvement discutée dans la section précédente. Elle exclut cependant d'entrée de jeu qu'une croyance que $P$ et une acceptation que non-P puissent simultanément figurer dans l'arrière-plan cognitif adapté d'une même délibération. En outre, cette norme n'implique pas que les éléments de l'arrière-plan adapté soient cohérents avec toutes les croyances de l'agent. Pour l'argument présent, il ne sera pas nécessaire d'aller jusqu'à supposer cette forme plus forte de cohérence avec les croyances.

Cohérence explicative dans l'arrière-plan adapté. Plusieurs auteurs ${ }^{19}$ ont soutenu qu'une croyance que $P$ doit être supportée par un réseau de croyances concernant la justesse de cet état, qui dans le cas présent se résume à des croyances concernant les évidences de l'agent en faveur de P. De la même manière, je supposerai ici qu'un élément donné dans l'arrière-plan cognitif adapté d'une délibération doit être supporté par un réseau d'autres éléments de l'arrière-plan cognitif adapté concernant la justesse de cet élément. Pour autant que ceux-ci soient des croyances n'ayant pas été fixées ni suspendues, en d'autres termes pour autant que ceux-ci ne soient pas des acceptations, cette contrainte n'est pas différente de celle s'appliquant aux croyances dans l'arrière-plan cognitif par défaut. Elle demande que certains éléments de l'arrière-plan cognitif adapté concernent les évidences de l'agent à propos des croyances en question. Pour les acceptations, on étendra cette contrainte aux raisons pratiques pouvant justifier la fixation ou la suspension d'une croyance, de sorte qu'une acceptation que $\mathrm{P}$ dans un contexte donné doit être accompagnée d'un réseau d'éléments de l'arrière-plan adapté, acceptations ou croyances, concernant soit les évidences en faveur de $\mathrm{P}$, soit les avantages pratiques de tenir $\mathrm{P}$ pour acquis dans le présent contexte.

La dérivation cognitiviste des normes de rationalité associées aux intentions utilise une prémisse supplémentaire concernant les sources légitimes d'évidences dans le cas particulier des croyances d'un agent à propos des actions qu'il accomplira intentionnellement lui-même, à savoir qu'un agent est justifié de croire qu'il fera A intentionnellement seulement s'il croit qu'il a l'intention de faire A ou s'il croit qu'il formera cette intention au moment opportun $^{20}$. Je ferai la même supposition concernant l'arrière-plan cognitif adapté: un agent est justifié de croire ou de tenir pour acquis qu'il accomplira l'action A dans une délibération donnée si et seulement s'il croit ou tient

19. Voir notamment Harman (1986) et les références sur les approches holistes des croyances dans (Schwitzgebel, 2006).

20. Voir les détails dans Roy (2006, chap. 6). 
pour acquis dans cette délibération qu'il a l'intention de faire A ou qu'il formera cette intention en temps et lieu.

Les normes d'agglomération systématique, de cohérence interne, de cohérence avec les croyances et de cohérence explicative des acceptations découlent toutes de ces trois normes générales s'appliquant à l'arrière-plan cognitif adapté des délibérations. Il est important de garder en tête que ces normes sont relatives aux contextes délibératifs.

\section{Une forme hybride de pragmatisme}

Dans cette section je développe une explication pragmatiste hybride des normes associées aux intentions sur la base du concept d'acceptation en contexte délibératif et des normes de rationalité lui étant associées qui ont été présentées dans la section précédente. Cette approche est pragmatiste au sens où, comme l'approche proposée par Bratman (à paraître), elle fait explicitement intervenir des normes de rationalité pratique. Elle fait cependant aussi intervenir des normes s'apparentant aux normes de rationalité théoriques associées aux croyances, d'où le qualificatif « hybride».

La section commence par une présentation de ce que j'appellerai le postulat pragmatiste hybride, reflétant le postulat cognitiviste moyen. Je présente ensuite les dérivations des normes associées aux intentions.

\subsection{Le postulat pragmatiste hybride}

L'idée clé derrière l'approche que j'explore ici est de supposer qu'avoir l'intention de faire A implique de tenir certains faits pour acquis dans certains contextes délibératifs. Plus précisément:

(PPH) Avoir l'intention de faire A implique de tenir pour acquis, dans les délibérations où cette intention est pertinente, que l'on fera $A$.

Cette formulation est délibérément proche du postulat cognitivisme moyen (PCM) énoncé plus haut, à la différence qu'il remplace la notion de croyance par celle d'acceptation, qui s'accompagne d'une clause de pertinence absente du $\mathrm{PCM}^{21}$.

La clause de pertinence a pour but de prévenir une surcharge de l'arrièreplan cognitif adapté qu'entraînerait une contrainte voulant qu'avoir l'intention de faire A implique accepter en délibération, peu importe laquelle, que l'on fera A. Certaines intentions ne sont clairement pas pertinentes dans certains contextes délibératifs. Pensons par exemple à un agent qui considère les différents menus du midi offerts à la cantine universitaire. Le fait qu'il ait, en outre, l'intention d'écrire une thèse de doctorat dans les prochains mois n'a vraisemblablement pas d'influence sur le plat qu'il choisira. En d'autres termes, on peut

21. Plusieurs auteurs ont proposé ou discuté des principes s'apparentant au PPH, notamment Harman (1976, p. 438), Bratman (1999, p. 32) et Wallace (2006, post-scriptum au chapitre 5). 
très bien imaginer que son intention d'écrire une thèse de doctorat n'est pas pertinente dans la délibération présente, et donc que ce fait ne figurera pas explicitement dans l'arrière-plan cognitif adapté à cette délibération.

Le but premier de ce postulat est de jeter une base suffisamment solide pour dériver les normes de rationalité associées aux intentions présentées plus haut, ce à quoi je m'applique à l'instant, tout en demeurant ouvert à la possibilité qu'un agent puisse avoir une réelle intention d'accomplir l'action $\mathrm{A}$ tout en demeurant dans le doute quant au fait qu'il parviendra à faire A. Cela n'est pas possible sous le postulat cognitiviste moyen : avoir l'intention de faire A implique, pour reprendre le vocabulaire de la section précédente, inclure la croyance que l'on fera A dans l'arrière-plan cognitif par défaut de l'agent, croyance qui est incompatible avec celle que, peut-être, l'agent ne parviendra pas à faire A. Dans une perspective pragmatiste hybride, au contraire, être dans le doute quant au fait que l'on fera A est parfaitement compatible avec l'acceptation, dans certains contextes, que l'on accomplira cette action.

\subsection{La dérivation des normes de rationalité associées aux intentions}

Je présente ici le détail de la dérivation des quatre normes de rationalité associées aux intentions que j'ai identifiées dans la Section 2. Les dérivations des normes de cohérence interne, de cohérence avec les croyances et d'agglomération ciblée sont relativement directes. Celle de la cohérence moyens-fin nous amènera cependant à introduire et à discuter assez longuement d'un principe additionnel de rationalité visant à contourner ce que j'appellerai le problème des acceptations incorrectes.

Cohérence interne. Cette norme est une conséquence directe du postulat pragmatiste hybride et de la norme de cohérence interne des acceptations: un agent ayant une intention dont le contenu est contradictoire violera du même coup, par le PPH, la norme de cohérence interne des acceptations. En replaçant cet argument contrapositif à l'endroit, on obtient que la cohérence interne des acceptations et le postulat pragmatiste hybride impliquent ensemble la cohérence interne des intentions.

Cohérence avec les croyances. Suivant le modèle de dernière dérivation, le but ici est de montrer qu'une violation de la norme de cohérence des intentions avec les croyances engendre une violation de la norme de cohérence des acceptations avec les croyances.

Supposons donc qu'un agent ait l'intention de faire A, que A ne puisse pas être accomplie si ses croyances s'avéraient et, assumant ici un principe de fermeture sous la conséquence logique, que ce fait figure également dans l'arrière-plan cognitif par défaut de cet agent. Pour engendrer la violation requise de la norme d'acceptation avec les croyances, il suffit de montrer qu'en général cette croyance et l'acceptation engendrée selon le postulat pragmatiste hybride par l'intention de faire A seront simultanément pertinentes dans certains contextes délibératifs. 
Prenons donc un contexte délibératif où tenir pour acquis que l'agent fera A a une influence significative sur l'issue de la délibération. Comme je l'ai mentionné plus haut, j'entends par là que la décision qui serait prise en tenant compte de cette acceptation diffère de celle qui serait prise si l'agent ne tenait pas pour acquis qu'il fera A. On remarquera que la croyance que A n'est pas réalisable implique la croyance que A ne sera pas réalisée, ou simplement que l'agent ne fera pas A. Manifestement, cette croyance est aussi pertinente pour la délibération présente : elle implique la condition plus faible que l'agent n'exclut pas la possibilité qu'il ne fera pas A, qui est équivalente au fait de ne pas croire qu'il fera A, fait qui, par hypothèse, influence de manière significative l'issue de la présente délibération. On a donc que l'acceptation que l'agent fera A et la négation de cette acceptation, ici sous forme d'une croyance qu'il ne fera peut-être pas A, sont présentes dans le même contexte délibératif, d'où la violation requise du principe de cohérence des acceptations avec les croyances.

Agglomération. On obtient sans effort l'agglomération ciblée une fois qu'on a montré que le pragmatisme hybride est en mesure de dériver la norme de cohérence interne des intentions et celle de cohérence avec les croyances. Il suffit en effet de montrer qu'une violation de la première engendre une violation d'une des secondes. Une violation de l'agglomération ciblée n'est cependant rien de plus qu'un cas où l'agent a l'intention de faire $\mathrm{A}$ et l'intention de faire B mais qu'il n'a pas l'intention de faire A et B et que, en outre, cette intention conjointe viole soit la norme de cohérence des intentions avec les croyances, soit la norme de cohérence interne des intentions. Tout ce qu'il importe de montrer est donc que ces deux normes sont bel et bien dérivables pour le pragmatisme hybride, ce qui a été fait à l'instant.

Cohérence moyens-fin. Supposons donc qu'un agent ait l'intention d'accomplir l'action F, qu'il croit qu'il ne peut accomplir cette action sans accomplir intentionnellement l'une des actions dans l'ensemble de moyens $\mathrm{M}$, mais qu'il n'a ni l'intention d'accomplir intentionnellement certaines de ces actions ni celle de former l'intention requise plus tard. Prenons une délibération où l'intention de faire $\mathrm{F}$ est pertinente au sens où le résultat de cette délibération est influencé de manière significative par la prise en compte que l'agent fera $\mathrm{F}$ dans cette délibération. On obtient, par le postulat cognitiviste, que l'agent tient pour acquis qu'il fera F. La croyance de l'agent qu'il ne peut faire $\mathrm{F}$ sans faire une action dans $\mathrm{M}$ est donc également pertinente pour cette délibération, parce qu'elle concerne le contenu d'une intention qui est prise en compte dans cette délibération. Par le principe de fermeture de l'arrière-plan cognitif sous la conséquence logique, on obtient que l'agent accepte dans cette délibération qu'il fera une des actions dans $\mathrm{M}$, acceptation qui, en l'absence de l'acceptation que l'agent a l'intention de faire une des actions dans $\mathrm{M}$, viole le principe de cohérence explicative de l'arrière-plan adapté. 
Pour mener cet argument à terme, il faut cependant montrer que l'agent ne tient pas pour acquis dans cette délibération qu'il a l'intention de faire une des actions dans M. Malheureusement, aucun principe avancé jusqu'ici ne nous permet de tirer cette conclusion, parce que les acceptations, tout comme les croyances, peuvent en principe être erronées, ou plutôt injustifiées. En d'autres termes, rien ne permet de conclure a priori qu'un agent n'ayant pas l'intention de faire une des actions de l'ensemble $\mathrm{M}$ ne tient pas à tort pour acquis qu'il possède tout de même cette intention.

La dérivation cognitiviste est aux prises avec une difficulté analogue, que je nommerai dans le contexte présent le problème des acceptations incorrectes. Comme Wallace (2003), j'endosse ici l'idée que cette difficulté ne peut pas être surmontée sans supposer qu'une telle acceptation incorrecte à propos de ses propres intentions est simplement irrationnelle ${ }^{22}$. Plus précisément, j'adopterai ici l'idée que dans les contextes où une certaine intention de faire $\mathrm{A}$ est ou serait pertinente, être correct à propos de cette intention, au sens de tenir pour acquis qu'on a l'intention de faire A si et seulement si l'on a effectivement l'intention d'accomplir cette action, est « une vertu pratique, devant être incluse parmi les traits et capacités qui nous rendent, d'une manière générale, efficaces dans la poursuite de nos buts ${ }^{23}$. En d'autres termes, tenir pour acquis qu'on a l'intention de faire $\mathrm{A}$ dans un contexte où cette intention est pertinente alors que l'on ne possède pas cette intention, et mutatis mutandis dans un contexte où l'on possède cette intention alors qu'elle serait pertinente, est irrationnel du point de vue de notre capacité générale d'arriver aux fins que nous décidons de poursuivre.

Un tel principe permet évidemment de contourner le problème des acceptations incorrectes. Si, dans l'argument ci-dessus, l'on suppose que l'agent en question est rationnel au sens où il se conforme au principe tout juste avancé, alors nous pouvons conclure que le fait qu'il n'a pas l'intention de faire une des actions dans $\mathrm{M}$ ne se trouve pas dans l'arrière-plan cognitif adapté de la délibération, ce qui nous permet bel et bien de conclure à une violation de la norme de cohérence explicative.

La version cognitiviste du principe tout juste avancé fut remise en question par Bratman (à paraître) sur la base de l'idée qu'il n'est pas toujours, pratiquement parlant, irrationnel d'avoir des acceptations incorrectes à propos

22. Wallace note, dans un contexte cognitiviste: «theoretical constraints on rational beliefs can get you as far as the belief that you intend to do [something]; to go beyond that, to a rational requirement that you form the [required means-intention], we need an additional principle [...].» (2003, p. 21).

23. Ce principe est adapté de celui proposé par Wallace pour sauver la dérivation cognitiviste de la cohérence moyens-fin : "in deliberative contexts where [means-end coherence] is relevant, [...] an executive virtue, to be included among the traits and capacities that make us, in general, effective in the pursuit of our goals ". Plus loin il précise cette idée en affirmant que ne pas être dans l'erreur à propos de ses propres intentions " is a strategy that enhances our ability to realize the broader aims that are given with our nature as deliberating agents ». (Wallace, 2006, p. 119). 
de ses propres intentions. Il note, que «se pencher soigneusement sur ce qu'on a l'intention de faire [...] est une activité qui demande temps et énergie, et il se peut très bien [qu'on] ait mieux à faire $»^{24}$. À titre d'exemple, il nous invite à imaginer un agent qui, un dimanche, croit qu'il a l'intention d'aller au magasin le vendredi suivant, alors qu'il a en fait l'intention d'y aller le jeudi. Cela n'est pas nécessairement irrationnel, soutient Bratman, parce qu'on peut très bien concevoir que, pour cet agent, "d'autres choses sont plus pressantes à ce moment [dimanche] ${ }^{25}$.

Il est important de voir que ce contre-exemple n'est pas décisif, ni pour le principe cognitiviste avancé par Wallace ni pour celui énoncé plus haut en termes d'acceptation, parce qu'il fait fi de la clause de pertinence. Dans l'exemple ci-dessus, le fait d'avoir une croyance erronée à propos de son intention de se rendre au magasin ne met pas en péril l'accomplissement des fins de l'agent pour autant que cette croyance n'est pas pertinente pour une délibération. Supposons, par exemple, que l'agent entende se rendre au magasin pour se procurer des articles de plein air dont il aura besoin pour ses prochaines vacances estivales, et qu'il délibère sur certains détails relatifs à ce congé en tenant pour acquis qu'il aura en main les articles qu'il entend se procurer. On peut très bien imaginer que les conclusions de cette délibération ne soient pas mises en cause par le fait que notre agent est dans l'erreur à propos de la journée à laquelle il entend se rendre au magasin, pour autant qu'il se procure à un certain moment les articles en question. Cette croyance fausse s'avérera cependant problématique si ce dernier la prend en compte en planifiant sa journée de vendredi. Dans ce contexte délibératif, l'argument selon lequel l'agent ne devrait pas réexaminer sa croyance erronée parce que d'autres choses sont plus pressantes ne semble pas tenir la route.

Cette dépendance du principe de rationalité des croyances d'un agent à propos de ses propres intentions est d'autant plus forte pour la version de ce principe qui concerne les acceptations en contextes délibératifs. Ce principe stipule, en effet, qu'une acceptation incorrecte d'un agent concernant ses propres intentions est irrationnelle dans les contextes où cette intention est pertinente, c'est-à-dire dans les contextes délibératifs dont l'issue dépend de manière significative de la prise en compte ou non du fait que cette intention sera réalisée. Le contre-exemple proposé par Bratman ne remplit pas cette condition, parce que, par hypothèse, tenir pour acquis ou non que l'agent se rendra au magasin vendredi, et non pas seulement qu'il se rendra au magasin tout court, n'a pas d'influence significative sur les délibérations considérées, c'est-à-dire sur ce qui est en l'occurrence plus pressant. Il est clair qu'une telle acceptation incorrecte dans les contextes où elle est réellement pertinente, par

24. Traduction libre de "to reflect carefully on all that one intends [...] is an activity that takes time and uses other resources, and one may well have better things to do " (à paraitre, p. 13).

25. Traduction libre de "other matters are more pressing right now» (Bratman, idem). 
exemple dans le cas où l'agent planifie la journée de vendredi, met en péril l'accomplissement de ce qui sera décidé lors de la délibération.

On notera également que le principe énoncé plus haut ne dépend pas du fait que la réalisation des actions choisies soit nécessairement compromise par la mise en jeu d'acceptations incorrectes à propos d'intentions pertinentes. Le principe réfère, en effet, à la capacité générale des agents à arriver à leurs fins, et non à l'actuelle réalisation de chacune de leurs décisions. De ce fait, ce principe ne condamne pas les acceptations incorrectes à propos d'intentions pertinentes parce qu'elles mettent systématiquement en cause la réalisation des fins de l'agent, mais plutôt parce qu'avoir de telles acceptations est, pour ainsi dire, une habitude non recommandable du point de vue de notre capacité générale d'arriver à nos fins. Dans ce contexte, l'existence potentielle de cas où il ne serait pas irrationnel d'avoir des acceptations incorrectes ne constitue pas une objection décisive puisque, pour réfuter ce principe, il faut plutôt montrer qu'en général avoir des acceptations incorrectes n'est pas irrationnel, une thèse qui, sur la base de l'exemple tout juste examiné, parait difficile à défendre.

Nous aurons l'occasion de revenir sur ce principe de rationalité lors de la discussion générale de l'approche pragmatiste hybride à la fin de cette section, mais il est pour l'instant important de remarquer que ce principe semble beaucoup plus adapté à la présente dérivation de la norme de cohérence moyens-fin que la version cognitiviste proposée par Wallace. Le principe de rationalité avancé par ce dernier, tout comme la version adaptée aux acceptations que j'utilise, est résolument du domaine de la rationalité pratique. Or les approches cognitivistes, par exemple chez Velleman (2003), sont fréquemment motivées par l'ambition d'expliquer l'ensemble des normes de rationalité pratique associées aux intentions à partir de principes exclusivement théoriques associés aux croyances. On réduirait de ce fait une partie importante du domaine de la rationalité pratique à celui de la rationalité théorique. De ce point de vue, invoquer, comme le fait Wallace, un principe relevant du domaine pratique pour justifier la cohérence moyens-fin constitue une dérogation importante à l'entreprise cognitiviste, et on est en droit de demander ce qui justifie une telle manœuvre. L'invocation d'un principe de rationalité pratique est au contraire des plus naturelles dans la perspective du pragmatisme hybride. En faisant intervenir les acceptations en contextes délibératifs plutôt que les croyances, on met d'ores et déjà en jeu des états chevauchant les domaines de rationalité pratique et théorique. Il n'est donc pas surprenant de voir apparaître dans la dérivation des normes de rationalité basées sur les acceptations des principes à saveur plutôt théoriques, par exemple la fermeture sous la conséquence logique de l'arrière-plan cognitif adapté, et un principe résolument pratique concernant les acceptations incorrectes à propos d'intentions. 


\subsection{Argument général en faveur du pragmatisme hybride}

Je veux ici examiner dans une perspective plus générale les deux principes ou postulats, le PPH et la norme liant intentions et acceptations à leur propos, sur lesquels se base la dérivation pragmatiste hybride. J'esquisserai tout d'abord un argument en deux étapes en faveur du postulat pragmatiste hybride, argument dont la seconde étape rejoint le principe de rationalité des acceptations à propos d'intentions. Ces considérations mettent en lumière l'unité de l'approche pragmatiste hybride.

On peut, selon moi, défendre le postulat pragmatiste hybride en deux étapes. La première fait intervenir le pouvoir explicatif de ce principe en tant que thèse constitutive de la théorie des intentions, et la seconde propose d'y accoler une force normative à " portée large ${ }^{26}$, essentiellement pragmatique.

Le postulat pragmatiste hybride permet non seulement de dériver les normes de rationalité associées aux intentions, mais il permet également de répondre à une question jusqu'à présent peu traitée dans les théories contemporaines des intentions, à savoir comment ces dernières parviennent à exercer leur «influence sur le raisonnement ${ }^{27}$. Il est généralement accepté que les intentions ne sont pas seulement des éléments passifs du paysage mental des agents rationnels, que leur rôle ne se limite pas à incarner le résultat des raisonnements pratiques des agents. Les intentions préalablement adoptées sont également vues comme des éléments actifs du processus de prise de décision, parce qu'elles imposent un «filtre d'admissibilité » aux options considérées et qu'elles "poussent l'agent à délibérer ${ }^{28}$ sur les moyens à prendre pour être satisfaites.

En interprétant le PPH comme une thèse constitutive de la théorie des intentions, c'est-à-dire en soutenant que ce postulat décrit le processus par lequel l'influence des intentions s'étend au raisonnement pratique, on arrive à mieux comprendre comment sont mis en jeu le filtre d'admissibilité et le déclenchement de délibérations sur les moyens à prendre. Sous le postulat pragmatiste hybride, l'imposition du filtre d'admissibilité devient une simple application des normes de cohérence interne et de fermeture sous la conséquence logique s'appliquant à l'arrière-plan cognitif adapté. La prise en compte d'une option qui exclurait la satisfaction de l'intention de faire A, intention en outre pertinente dans cette délibération, reviendrait à considérer possible que l'action A ne soit pas accomplie, en contradiction directe avec l'acceptation relative à l'intention de faire A. De la même manière, la mise en lumière d'une incohérence

26. Je traduis ici l'expression "wide scope » mise de l'avant principalement dans les travaux de Broome, par exemple dans (1999).

27. Je traduis ici de manière assez libérale le terme "reasoning-centered commitment", proposé par Bratman (1986).

28. Je traduis ici "filter of admissibility " et "trigger deliberation ", la première expression venant directement des travaux de Bratman (1986) et la seconde adaptée de ces derniers dans (Roy, 2008) et (van Hees et Roy, 2008). 
moyens-fin dans la structure intentionnelle d'un agent, par exemple lors d'un raisonnement analogue à celui esquissé dans la dérivation de cette norme, peut agir comme élément déclencheur d'une délibération à propos des moyens de satisfaire l'intention qui pose problème.

À cet argument invoquant le pouvoir explicatif du PPH s'ajoute un argument normatif invoquant la visée générale des intentions. L'idée est ici, en premier lieu, de souligner que les intentions d'un agent visent, en général, le " contrôle des actions dans l'accomplissement de leur contenu » et que, de ce fait, elles «font partie des traits et capacités qui nous rendent, de manière générale, efficace dans la poursuite de nos buts ${ }^{29}$. » En couplant cette idée au postulat pragmatiste hybride, on obtient une réponse générale à la question de savoir pourquoi les intentions devraient jouer le rôle actif qu'on leur suppose ou, en d'autres termes, une réponse à la question de savoir pourquoi les agents devraient adopter le postulat pragmatiste hybride. Ce principe constitue une manière privilégiée pour un agent de rendre ses intentions conformes à leur visée générale.

On notera au passage que cet argument normatif est, dans les termes de Broome (1999), «à portée large »; il vise à montrer que les agents devraient se conformer au postulat pragmatiste hybride, et non qu'un agent ayant l'intention de faire A a, de ce fait, de bonnes raisons (pratiques) d'accepter dans les contextes délibératifs pertinents qu'il fera A. La clause normative « devrait » n'est pas détachable ${ }^{30}$.

La visée essentiellement pragmatique attachée aux intentions est naturellement mise en œuvre dans la délibération à travers des états mentaux qui sont, eux aussi, sensibles aux considérations pratiques, soit les acceptations en délibérations, et ce, d'une manière qui unifie la justification du PPH et celle du principe de rationalité des acceptations à propos d'intentions utilisé dans la dérivation de la cohérence moyens-fin ${ }^{31}$. Le souci général d'arriver à ses fins que j'ai invoqué pour justifier ce principe est, en effet, le même que celui invoqué pour décrire la visée des intentions. Vus sous cet angle, le PPH et le principe de rationalité des acceptations à propos des intentions tirent leur force normative de la même idée de rationalité pratique en terme de capacité à réaliser ses fins.

29. Traductions libres de «coordinated control of action that achieves what is intended » (Bratman, à paraître, p. 33, italique dans l'original) et de "among the traits and capacities that make us, in general, effective in the pursuit of our goals" (Wallace, 2006, p. 118).

30. J'emprunte également le terme "détachable" à Broome (1999).

31. Cette thèse supporte également l'idée mentionnée dans la section 5.2, à savoir que le pragmatisme hybride propose une justification plus uniforme que celle de Wallace des normes de rationalité associées aux intentions. En fait, la stratégie argumentative de Wallace semble tellement mieux adaptée au concept d'acceptation qu'à celui de croyance qu'on est en droit de se demander pourquoi ce dernier n'a pas fait le saut définitif vers le pragmatisme hybride, plutôt que de rester dans une perspective cognitiviste. 
Il est important de remarquer que même si la force normative pragmatique du PPH est intimement liée à la thèse constitutive selon laquelle ce postulat permet d'expliquer comment les intentions influencent le raisonnement pratique, la seconde thèse est indépendante de la première. Une approche alternative aurait tout aussi bien pu accoler à l'argument constitutif une force normative de type cognitiviste, sur la base de normes de rationalité théoriques.

Dans ce contexte, on peut être tenté de reprocher au pragmatisme hybride sa position mitoyenne. En invoquant à la fois une série de principes apparemment théoriques régissant la cohérence de l'arrière-plan cognitif adapté et un principe général pragmatiste, le pragmatisme hybride laisse tomber une thèse ambitieuse, présente tout aussi bien chez de nombreux cognitivistes que dans l'approche strictement pragmatiste de Bratman (à paraître), selon laquelle les normes de rationalité associées aux intentions découlent de normes relevant uniquement soit du domaine théorique, soit du domaine pratique.

Que le pragmatisme hybride n'ait pas le panache d'une approche purement cognitiviste ou purement pragmatique est, à mon point de vue, un avantage plutôt d'un inconvénient. En invoquant des normes plutôt théoriques s'appliquant à l'arrière-plan cognitif adapté pour justifier les normes de cohérence interne, de cohérence avec les croyances et d'agglomération ciblée, le pragmatisme hybride rend justice à la connotation théorique de ces normes, c'est-à-dire au fait qu'elles semblent toutes destinées à éviter certaines contradictions logiques dans la structure intentionnelle des agents, connotation qui est perdue dans l'approche purement pratique de Bratman. D'un autre côté, en plaçant un principe pragmatiste au cœur de la justification de la norme de cohérence moyens-fin, le pragmatisme hybride fait de cette norme une norme fondamentalement pratique, ce qui semble non seulement plus intuitif mais rejoint également une longue tradition en théorie de la rationalité instrumentale, remontant au moins jusqu'à Kant. En somme, le pragmatisme hybride argumente peut-être d'une manière moins éclatante que le pragmatisme ou le cognitivisme pur, mais d'une manière qui semble mieux correspondre à nos intuitions quant aux sources normatives des normes de rationalité associées aux intentions.

\section{Conclusion}

Dans cet article, j'ai exploré une approche de la justification des normes de rationalité associées aux intentions, le pragmatisme hybride, qui utilise la notion d'acceptation en délibération. J'ai montré que cette approche constitue une alternative plausible à celles proposées jusqu'à présent dans la littérature en ce que, d'un côté, elle permet de dériver lesdites normes d'une manière qui évite certains problèmes de l'approche cognitiviste pure et que, de l'autre, elle rend justice à nos intuitions concernant les sources normatives de ces normes. J'ai soutenu de plus que le pragmatisme hybride offre une explication naturelle du mécanisme par lequel les intentions influencent la délibération. 
J'ai dû, en cours de route, laisser de côté beaucoup de questions auxquelles l'approche pragmatiste hybride devra éventuellement répondre. La plus criante concerne la justification des principes de rationalité associés à l'arrièreplan cognitif adapté aux délibérations, qui fut complètement passée sous silence. La notion de pertinence pour une délibération devra également être raffinée, tout comme les détails des arguments généraux en faveur du pragmatisme hybride que j'ai esquissés dans la section 5.3. Ces questions ne doivent pas, selon moi, être vues comme des carences. Plutôt, on doit les considérer comme des incitations à explorer davantage une justification des normes de rationalité associées aux intentions qui sort des sentiers battus par le cognitivisme et le pragmatisme pur, et qui, de ce fait, nous force à repenser le paysage traditionnel en philosophie analytique de l'action.

\section{Remerciements}

Je désire avant tout remercier Michael Bratman, dont les commentaires ont guidé le développement des idées présentées ici, de leurs premiers balbutiements jusqu'à la toute dernière version de cet article. Cet article doit également beaucoup à Martin van Hees et Johan van Benthem, ainsi qu'aux discussions avec Facundeo Alonso, Randall Harp, Jennifer Morton et Sarah Paul. Merci enfin à Renée Bilodeau de m'avoir donné la chance de publier ce texte en français, et une pensée à Nicolas Kaufmann, qui a exercé une influence profonde sur mon développement intellectuel.

Les travaux présentés ici ont été réalisés grâce au soutien financier du Fonds québécois de la recherche sur la société et la culture (bourse \# 93233) et du Conseil de la recherche en sciences humaines du Canada (bourse \# 7522006-0345). Ils ont été réalisés en grande partie lors de mes études doctorales à l'Institute for Logic, Language and Computation de l'Université d'Amsterdam.

\section{Bibliographie}

Alonso, F. Shared Intention, Reliance, and Interpersonal Obligations. Thèse doctorale, Stanford University, 2008.

Bratman, M. Intention, Plans and Practical Reason. Harvard UP, 1987.

- Faces of Intention; Selected Essays on Intention and Agency. Cambridge UP, 1999.

__. «Practical Reasoning and Acceptance in a Context. » Nô̂s, 1991. Reproduite dans (Bratman, 1999, p. 15-34). Les références dans le texte sont à cette seconde édition.

__. «Intention, Belief, Practical, Theoretical ». À paraître dans J. Timmerman, J. Skorupski, et S. Robertson (eds.), Spheres of Reason, Oxford UP.

Broome, J. « Normative Requirements ». Ratio (new series), XII (4), décembre 1999.

Cohen, L. J. «Belief and acceptance». Mind, 98 (391) : 367-389, juillet 1989.

Engel, P. «Believing, Holding True, and Accepting ». Philosophical Explorations, 1 (2): 140-151, 1998.

Grice, H. P. «Intention and Uncertainty ». Dans Proceedings of the British Academy, volume 57, 1971, Oxford UP. 
Harman, G. Change in View. MIT Press, 1986.

_. "Practical Reasoning ». Review of Metaphysics, 29 (3) : 431-463, 1976.

Holton, R. "Deciding to Trust, Coming to Believe». Australasian Journal of Philosophy, 72 (1), 1994.

Pollack, M. E. «Overloading Intentions for Efficient Practical Reasoning ». Nô̂s, 25 (4) : 513-536, 1991.

Roy, O. Thinking Before Acting : Intentions, Logic, Rational Choice. Thèse doctorale, Universiteit van Amsterdam, 2008.

Schwitzgebel, E. Entrée « Beliefs » dans The Stanford Encyclopedia of Philosophy, août 2006. URL http ://plato.stanford.edu/. E. Zalta (ed.).

Setiya, K. Reasons Without Rationalism. Princeton UP, 2007.

- "Cognitivism about Instrumental Reason ». Ethics, à paraître.

Shah, N. et J. D. Velleman. Doxastic Deliberation. The Philosophical Review, 114 (4): 497-534, 2005. La pagination utilisée dans le texte provient d'une version préliminaire téléchargée sur le site web de J.D. Velleman.

Velleman, J. D. Self to Self. Cambridge UP, 2005.

—. "What Good is a Will ? ». Dans Anton Leist (ed.), Action in Context, Walter de Gruyter, 2007.

van Hees, M. et O. Roy. "Intentions and Plans in Decision and Game Theory » dans Bruno Verbeek (ed.), Reasons and Intentions. Ashgate. 2008.

Wallace, R. J. « Normativity, Commitment, and Instrumental Reason ». Philosophers' Imprint, 1 (3) : 1-26, 2003. . Normativity and the Will. Oxford UP, 2006.

Williams, B. "Ethical Consistency ». In Problems of the Self. Cambridge UP, 1973.

Yaffe, G. "Trying, Intending, and Attempted Crimes". Philosophical Topics, 32 (1-2), 2004. 